\title{
Nosocomial infection of extensively drug-resistant Myroides odoratimimus in a Turkish hospital
}

\author{
Unsal Savci $^{1}$, Baris Eser ${ }^{1}$, Mustafa Sungur ${ }^{2}$, Mustafa Sahin ${ }^{1}$, Nezahat Kosar ${ }^{3}$, Djursun \\ KARASARTOVA $^{4}$, Ayse Semra GURESER ${ }^{5}$, Aysegul Taylan-Ozkan ${ }^{6}$, Selahattin \\ Caliskan $^{7}$, Serap Suzuk Yildiz ${ }^{8}$, Fatma Bayrakdar ${ }^{9}$, Yasemin Numanglu Cevik ${ }^{8}$, Coskun \\ Kaya $^{10}$, Derya Yapar ${ }^{11}$, and Ozlem Akdogan ${ }^{12}$ \\ ${ }^{1}$ Hitit University \\ ${ }^{2}$ Eskişehir State Hospital \\ ${ }^{3}$ Hitit University, Turkey \\ ${ }^{4}$ Hitit Universitesi \\ ${ }^{5}$ Hitit University, Faculty of Medicine \\ ${ }^{6}$ Hitit University, Turkey \\ ${ }^{7}$ Reyap Hospital, Department of Urology \\ ${ }^{8}$ Public Health Institutions of Turkey \\ ${ }^{9}$ Public Health Institutions of Turkey, Ankara, Turkey. \\ ${ }^{10}$ Eskisehir City Hospital, Department of Urology \\ ${ }^{11}$ Hitit University Faculty of Medicine,Turkey. \\ 12 Hitit University Faculty of Medicine,Turkey.
}

October 17, 2020

\begin{abstract}
Objectives: Myroides spp. is an environmental pathogen and causes disease in immunocompromised patients. In this study, we report an outbreak of urinary tract infections caused by M. odoratimimus in a university hospital in Turkey. Methods: A total of $25 \mathrm{M}$. odoratimimus strains isolated from the clinical samples of 20 patients in our intensive care units and clinics were included in the study. Phenotypic and genotypic identification of isolates was performed using conventional methods, VITEK@-2 automated identification system, Matrix Assisted Laser Desorption/Ionisation-Time of Flight Mass Spectrometry and 16S-RNA Microbial Diagnosis methods. In addition, Repetitive Extragenic Palindromic Elements (REP) PCR Assay method was applied for molecular epidemiological analysis. Results: All cases were diagnosed with nosocomial urinary tract infection, except for one case diagnosed with nosocomial bacteraemia. One of the M. odoratimimus isolates was sensitive to piperacillin/tazobactam (MIC: [?]4 $\mu \mathrm{g} / \mathrm{ml}$ ) and one isolate was moderately sensitive to cefepime (MIC: $16 \mu \mathrm{g} / \mathrm{ml}$ ). Other M. odoratimimus isolates were resistant to the tested antibiotics of beta lactams, monobactams, carbapenems, aminoglycosides, fluoroquinolones and sulphonamides. When 10 isolates were evaluated with the REP PCR method, DNA finger print similarities were visually determined and there was a similar DNA pattern among them. Myroides source was not detected in environmental samples. Conclusion: Clinicians should consider that Myroides spp. isolates with multiple and broad-spectrum drug resistance may be a serious nosocomial pathogen like Pseudomonas aeruginosa or Acinetobacter baumannii. In order to choose the best treatment regimen, this atypical pathogen needs to be quickly identified and antibiotic susceptibility tests performed.
\end{abstract}

\section{Hosted file}

Main text.pdf available at https://authorea.com/users/368008/articles/487263-nosocomial- 
infection-of-extensively-drug-resistant-myroides-odoratimimus-in-a-turkish-hospital

\section{Hosted file}

table 1.pdf available at https://authorea.com/users/368008/articles/487263-nosocomialinfection-of-extensively-drug-resistant-myroides-odoratimimus-in-a-turkish-hospital

\section{Hosted file}

Table 2.pdf available at https://authorea.com/users/368008/articles/487263-nosocomialinfection-of-extensively-drug-resistant-myroides-odoratimimus-in-a-turkish-hospital

\section{Hosted file}

Table 3.pdf available at https://authorea.com/users/368008/articles/487263-nosocomialinfection-of-extensively-drug-resistant-myroides-odoratimimus-in-a-turkish-hospital

\section{Hosted file}

Table 4.pdf available at https://authorea.com/users/368008/articles/487263-nosocomialinfection-of-extensively-drug-resistant-myroides-odoratimimus-in-a-turkish-hospital

\section{Hosted file}

Table 5. The incidence of Myroides spp. infections by months (1).pdf available at https: //authorea.com/users/368008/articles/487263-nosocomial-infection-of-extensively-drugresistant-myroides-odoratimimus-in-a-turkish-hospital

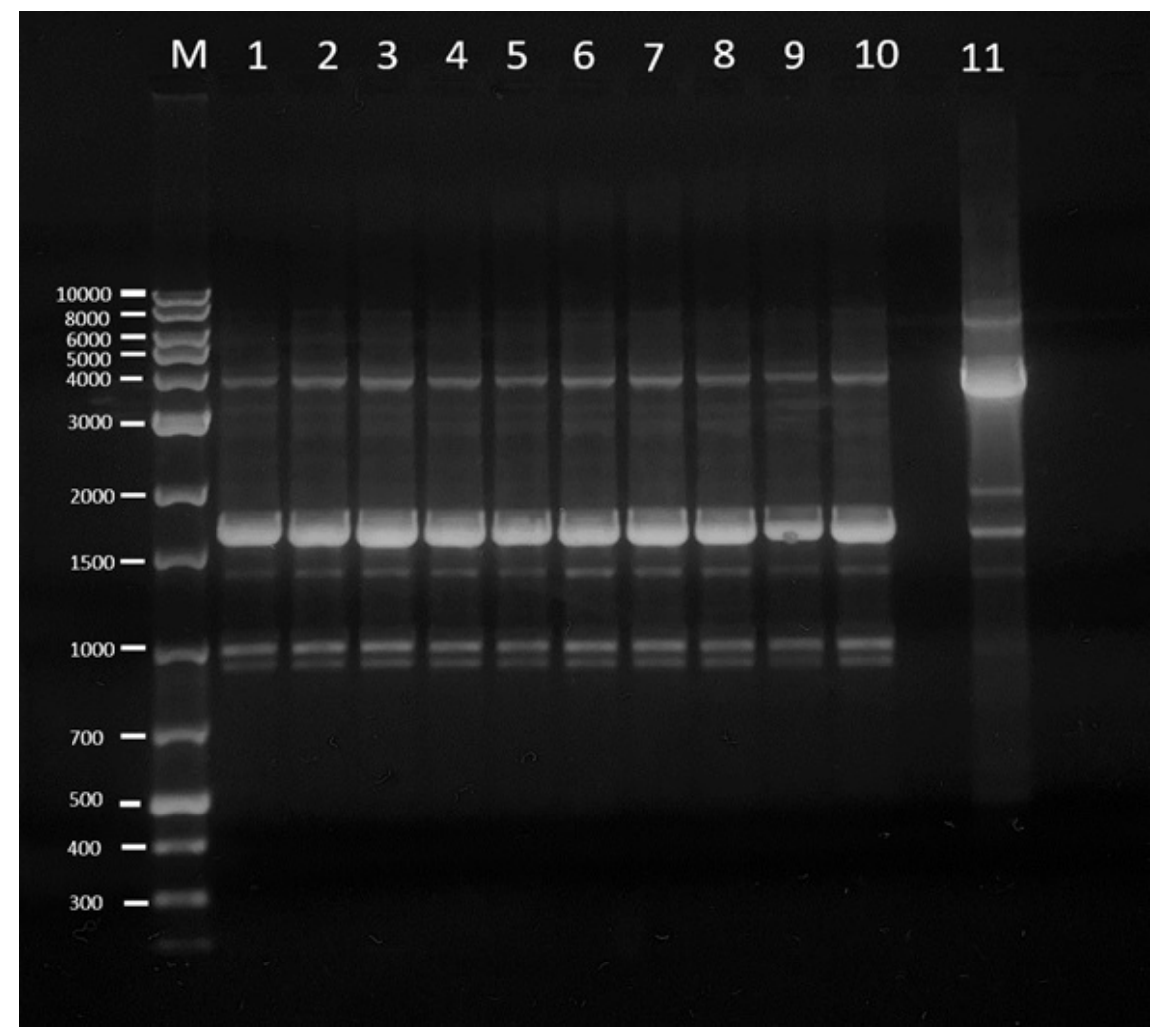

